\title{
(C) OPEN ACCESS \\ Home palliative care works: \\ but how? A meta-ethnography of the experiences of patients and family caregivers
}

\author{
Vera P Sarmento, ${ }^{1,2}$ Marjolein Gysels, ${ }^{1,3}$ Irene J Higginson, \\ Barbara Gomes ${ }^{1,4}$
}

- Additional material is published online only. To view please visit the journal online (http://dx.doi.org/10.1136/ bmjspcare-2016-001141).

'Department of Palliative Care, Policy and Rehabilitation, King's College London, Cicely Saunders Institute, London, UK

${ }^{2}$ Department of Medicine, Hospital do Espírito Santo de Évora, Évora, Portugal ${ }^{3}$ Centre for Social Science and Global Health, University of Amsterdam, Amsterdam, The Netherlands

${ }^{4}$ Faculty of Medicine, University of Coimbra, Coimbra, Portugal

\section{Correspondence to}

Dr Vera P Sarmento, Department of Medicine, Hospital do Espírito Santo de Évora, Serviço de Medicina 2, Lg. Do Sr. da Pobreza, s/n, Évora 7000-811, Portugal;

verapsarmento@gmail.com

Received 15 March 2016 Revised 14 December 2016 Accepted 30 January 2017 Published Online First 23 February 2017

\section{(a) CrossMark}

To cite: Sarmento VP, Gysels M, Higginson IJ, et al. BMJ Supportive \& Palliative Care 2017;7:390-403.

\section{ABSTRACT}

Objective To understand patients and family caregivers' experiences with home palliative care services, in order to identify, explore and integrate the key components of care that shape the experiences of service users.

Methods We performed a meta-ethnography of qualitative evidence following PRISMA

recommendations for reporting systematic

reviews. The studies were retrieved in 5 electronic databases (MEDLINE, EMBASE, Psyclnfo, BNI, CINAHL) using 3 terms and its equivalents ('Palliative', 'Home care', 'Qualitative research') combined with 'AND', complemented with other search strategies. We included original qualitative studies exploring experiences of adult patients and/or their family caregivers ( $\geq 18$ years) facing life-limiting diseases with palliative care needs, being cared for at home by specialist or intermediate home palliative care services.

Results 28 papers reporting 19 studies were included, with 814 participants. Of these, 765 were family caregivers and $90 \%$ were affected by advanced cancer. According to participants' accounts, there are 2 overarching components of home palliative care: presence (24/7 availability and home visits) and competence (effective symptom control and skilful communication), contributing to meet the core need for security. Feeling secure is central to the benefits experienced with each component, allowing patients and family caregivers to focus on the dual process of living life and preparing death at home.

Conclusions Home palliative care teams improve patients and caregivers experience of security when facing life-limiting illnesses at home, by providing competent care and being present. These teams should therefore be widely available and empowered with the resources to be present and provide competent care.

\section{BACKGROUND}

Palliative care provides high-quality care in any setting where patients with lifethreatening illnesses are cared for, being therefore considered as a human right. ${ }^{12}$ Receiving such care at home, according to patients and families' preferences, ${ }^{3}$ allows people to live in their own environment, while preserving the best quality of life possible. ${ }^{4}$ In the context of ageing populations with growing palliative care needs, ${ }^{5}$ and patients spending the majority of time being cared for at home, ${ }^{6}$ home care has become a policy priority. ${ }^{7}$ A Cochrane review ${ }^{8}$ showed that home palliative care services help meet preferences for being cared for at home, by doubling the odds for death at home while decreasing symptom burden. However, this review also found that the interventions differed considerably in the 23 studies identified. Such variability is inherent to the nature of a complex intervention such as home palliative care, which comprises the provision of holistic care usually by a multidisciplinary team. ${ }^{4}$ Therefore, the more than 1900 home palliative care teams existent in European countries are expected to provide different models of care, reflecting complexity and context diversity.

In the light of this variability, it is not well understood how home palliative care services benefit patients and their families. An integrative review of 17 studies of family caregivers' existential concerns identified loneliness, insecurity and responsibility as important concerns. ${ }^{10} \mathrm{~A}$ review of patients' and caregivers' satisfaction with palliative care services ${ }^{11}$ found that the perceived benefits of 
home palliative care included access to 24/7 support and symptom control. Evidence from the Cochrane review on home palliative care ${ }^{8}$ also suggests that $24 / 7$ availability might be an effective component of home palliative care.

Synthesising the qualitative evidence of the experiences of patients and family caregivers with home palliative care services will shed light on the role of the different components of the intervention. ${ }^{12}$ Albeit challenging, the identification of these key components, together with an understanding of how they influence the experiences of patients and families, could be useful to research, by informing the design of interventions to be tested in the future. It could also influence clinical practice and health policy, by shedding light on the minimum standards needed to provide home palliative care. ${ }^{13-15}$

In this study, we aimed to understand patients and family caregivers' experiences with home palliative care services in order to:

1. Identify the key components of home palliative care that shape the experiences of patients and family caregivers;

2. Explore the mechanisms of action of the identified key components according to patients' and family caregivers' experiences;

3. Integrate the findings into a model of patients' and family caregivers' experiences with home palliative care services.

\section{METHODS}

\section{Study design}

In order to ensure transparency and identify all relevant studies, we systematically reviewed published and unpublished literature, following a protocol designed and piloted by the team (presented as an online supplementary file) and according to PRISMA recommendations. ${ }^{16}$

To synthesise the studies, we chose an interpretative method of analysis in order to understand how home palliative care services work through the lenses of patients and caregivers. Meta-ethnography is a systematic method for synthesising qualitative evidence, that was first described by Noblit and Hare, ${ }^{17}$ in 1988. Since then, it has been successfully applied onto the healthcare field. ${ }^{18-20}$ This method consists of identifying and translating key concepts between studies, taking into account the context in which these concepts were obtained. At the end of the process, the meta-ethnographic analysis leads to a reciprocal, refutational and/or lines-of-argument synthesis. ${ }^{17}$

Given that meta-ethnography is a complex undertaking, we formed a multidisciplinary team comprising a range of different clinical and research experiences, including: a medical doctor with experience in palliative care research and clinical end-of-life care (VPS, first reviewer); a senior anthropologist and palliative care researcher with a background of linguistics and extensive experience in qualitative research and meta-ethnography (MG, second reviewer); a senior palliative care researcher with a psychology background and expertise in home palliative care research (BG, third reviewer). IJH was involved in the protocol development and overview of the study.

\section{Identification of studies}

Between September and November 2013, we searched the five major healthcare bibliographic databases (MEDLINE, EMBASE, CINAHL, PsycInfo, BNI) using text words and $\mathrm{MeSH}$ headings to capture three term groups ('Palliative', 'Home care', 'Qualitative research') combined with the operator 'AND' (electronic search strategies are presented in an online supplementary file). We have recorded the search results in an EndNote file and removed the duplicates.

The identification of qualitative studies through electronic search is traditionally challenging. ${ }^{21}$ Therefore, we complemented our search with other methods such as: hand-search of relevant publications, screening of references and citation search of relevant reviews and included studies, experts consultation and screening of conference proceedings and abstracts (non-electronic search strategies are presented in an online supplementary file).

\section{Selection of studies}

We considered the studies for inclusion through titles and abstracts screening, followed by full-text screening, according to the inclusion and exclusion criteria detailed in table 1 . We defined family caregivers as non-paid lay caregivers related by blood or friendship. Components of care were defined as the elements that build up a complex intervention, including behaviours, parameters of behaviours and methods of organising and delivering those behaviours. ${ }^{22}$ Mechanisms of action refer to how the components of care relate to each other in order to produce the outcome (ie, change in status of health and welfare of individuals or populations confidently attributable to the antecedent care). ${ }^{22}{ }^{23}$ To define home palliative care and distinguish generalist from intermediate or specialist services, we applied the criteria used by Gomes $e t a l^{8}$ in the Cochrane review (presented in an online supplementary file).

When needed, we contacted the authors of potentially relevant studies to ask for missing information. Finally, we discussed the inclusion of more than $10 \%$ of all full-text screened references within the team.

\section{Quality assessment}

To assess the quality of each study, we used a modified version of the CASP (Critical Appraisal Skills Programme) criteria, based on the work developed by Campbell et al ${ }^{1924}$ (detailed in the Protocol presented as online supplementary file). We answered the main CASP questions as yes/partially/no, covering issues related to methodological clarity, data collection, 
Inclusion criteria

Original research using and reporting qualitative methodologies

English, Portuguese, Spanish, French and Italian

Adult patients (aged 18+) with a life-limiting diagnosis and palliative care needs and/or their family caregivers being cared for at home

Specialist or intermediate palliative care provided at home*

Relevant findings for the identification of key components shaping the experiences of participants, the experiences shaped by these key components and mechanisms of action explaining these relationships
Exclusion criteria

Study does not report qualitative methods for data collection and analysis Any other language

Participants other than adult patients and/or their family caregivers (eg, voluntary caregivers)

Generalist home care (including end-of-life home care), intervention not sufficiently described, or experiences with specific components of care

Findings not related to the objectives of the review, or insufficiently informative results

${ }^{*}$ According to the criteria used by Gomes et $a l^{\beta}$ to define home palliative care and distinguish between specialist and intermediate services (presented in an online supplementary file).

analysis and reporting of results. The first reviewer performed the quality assessment, reported the results in a table and discussed these with the other reviewers. We did not exclude studies based on their quality assessment, as there is no evidence that this improves the quality of the review ${ }^{25}$ while it may exclude studies with low reporting quality but relevant results. ${ }^{19}$ The results of this appraisal were used to make sense of the general quality of the studies included and to identify potential pitfalls in the reporting that could influence the results of the review.

\section{Analysis}

We followed the steps of meta-ethnography described by Noblit and Hare, ${ }^{17}$ which involve five interactive stages:

1. Reading and re-reading the studies, and recording the following information in a predesigned extraction form, in a Microsoft Office Excel file: general characteristics, methods, participants, home palliative care services, results (themes, quotes and explanatory theories). At this stage, we concluded that the results of the studies seemed to agree in the overall understanding of the experiences of patients and caregivers with home palliative care services.

2. Through the process described above, we separately identified key components and experiences, followed by a discussion among the team. For this step, we used different methods in an interactive way, in order to take into account the whole of the findings. Hence, we summarised the table developed in the first stage and mapped the emerging experiences, components of care and their relationships, aiming to understand how the results of the studies related to each other.

3. We were then able to determine that the studies had reciprocal (similar) results (in contrast with refutational findings). At this point, we compared the concepts and maps obtained by each researcher, and agreed on the emerging experiences and key components of home palliative care.

4. By going back to the studies and data extraction forms, we translated the key components and experiences between the studies, ensuring that no relevant or contradictory findings were being ignored. This translation was reflected in a reciprocal translation table (presented in an online supplementary file).

5. After completing the stages above, we discussed the interpretations entailed in the results of the review. We identified first, second and third-order constructs by synthesising the reciprocal translation table in a reciprocal translation synthesis. A new level of interpretation was obvious at this point, with all components of care contributing to and explaining the same experience. Therefore, we obtained a lines-of-argument synthesis, which was then represented in a simplified conceptual model of the experiences of patients and caregivers with home palliative care.

\section{RESULTS}

\section{Identification and selection of studies}

We identified 4150 papers through the electronic search and after removing duplicates. From these, 3080 references were excluded in the screening of titles and abstracts. We retrieved the full text of the 1070 remaining references except for seven that we could not find despite attempts to contact the authors and the support of the library services. These are presented in an online supplementary file. From the remaining 1063 references, 1032 were excluded after full-text screening, resulting in 40 papers reporting 30 studies, from which 2 studies were identified through other sources (citation search and reference checking). We then read and re-read all 30 studies several times. Eleven studies and one paper were excluded at this phase because: seven studies reported experiences specifically with generalist home palliative care services; four studies presented a small amount of relevant results; one paper was about bereavement support only. Finally, 28 papers reporting 19 studies were included (list in an online supplementary file). All steps of the identification and selection of studies with reasons for exclusion are presented in a PRISMA flow chart (see online supplementary file). There was no specific order to read the studies. Initially, all studies were analysed to the same extent, and given the same 
importance. In order to ensure trustworthiness, all the findings reported by the studies were included in the first stage. Nonetheless, as the review process developed, some studies were found to present more comprehensive results that were cornerstone to the understanding of the experiences with home palliative care.

\section{Characteristics of included studies}

Table 2 presents the main characteristics of the 19 included studies. Eleven studies were undertaken in Sweden, four in the UK and one in Denmark. Three studies were from non-European countries (two from the USA and one from Australia). The 19 studies included data collected in semistructured or unstructured interviews in 14 cases (face-to-face or by telephone) and in 5 cases through other methods (structured questionnaires with open-ended questions in 4 studies and analysis of a written diary in 1 study). One of the Swedish studies was a secondary analysis of data collected in three of the included studies. Only one study was published before the year 2000 and four were published from 2011 onwards.

The 19 studies included 814 participants from which the majority were family caregivers $(n=765$, 94\%), whom participated in 17 of the 19 studies. Three studies alone included more than two-thirds of all caregivers $(n=522,68 \%)$. There was gender information for 680 caregivers, from whom 416 were women (61\%). About one-third of participant caregivers (237) were active carers at the time of the study, while 528 were bereaved caregivers. From all patients under home palliative care services $(\mathrm{n}=801)$, $724(90 \%)$ suffered from advanced cancer, while 77 (10\%) were diagnosed with advanced non-malignant diseases.

Nine studies sampled participants from specialist services, two from intermediate services and eight studies did not provide enough information to make the distinction between intermediate and specialist care. All services provided home visits, 16 included hands-on practical care, from which 4 provided advanced technological support (ie, interventions that are usually seen as institution-delivered, eg, blood transfusions, syringe drivers). Fifteen studies were conducted in services with 24 hours a day, 7 days a week (24/7) availability to be contacted, and seven visited patients at home out-of-hours $(\mathrm{OOH})$.

Overall, the studies had an acceptable methodological quality (see table 3), with only one study meeting less than half of the criteria (study 18journal letter) and 12 studies meeting 10 or more criteria. Less than half of the studies $(n=7)$ had an identified theoretical framework.

Interestingly, a relationship between the quality of the studies and the usefulness of their findings to the review was found. By reporting methods and findings thoroughly, and describing comprehensively the analyses and interpretations, higher quality studies better enlightened the understanding of experiences with home palliative care.

\section{Reciprocal comparative synthesis: key components of home palliative care}

We found that the results of the studies were reciprocally translatable. Although the experiences of patients and caregivers differed, the same care components seemed to meet their different needs. Patients and caregivers worked as dyads, with the suffering of one increasing the distress of the other.

Four components of the home palliative care teams contribute decisively to people's experiences of care: availability, home visits, effective symptom control and effective communication skills (second-order constructs, table 4), as illustrated by the quote below.

[What were the best aspects of hospital-based [home] palliative care?] 'The professionalism including experience and accessibility 24 hours a day. These two aspects have equal importance and cannot be ranked'. (caregiver, study 17)

These components can be grouped according to the meaning that patients and caregivers assign to them. A team that was available around the clock to be contacted, and that visited at home, was seen as being present for support. If the team effectively managed symptoms and communicated skilfully, it was understood by participants as being competent. Presence and competence were therefore identified as the third-order constructs (table 4). This reciprocal translational synthesis is presented in table 4 with illustrating quotes.

\section{Presence}

The home palliative care teams had a crucial role in providing a sense of being accompanied instead of being left alone in the difficult situation of dealing with an advanced life-limiting disease at home. To service users, this meant that professional help was easily accessed and that they were visited at home. Broadly, a team that was present yielded a sense of security and facilitated management of care at home.

When being able to get support by the team $24 / 7$, patients and caregivers' experience of security at home was enabled, as this meant that there was always someone knowledgeable available to give advice, who represented a better alternative than other immediate support (eg, calling an ambulance).

This availability decreased the uncertainty of the situation and facilitated trust in the team's competence and resources to meet their needs (table 4, Q1-Q4). $\mathrm{OOH}$ support could be exclusively telephonic or include availability for home visits if needed, though it was unclear if the experience of security was any different with these two models. 


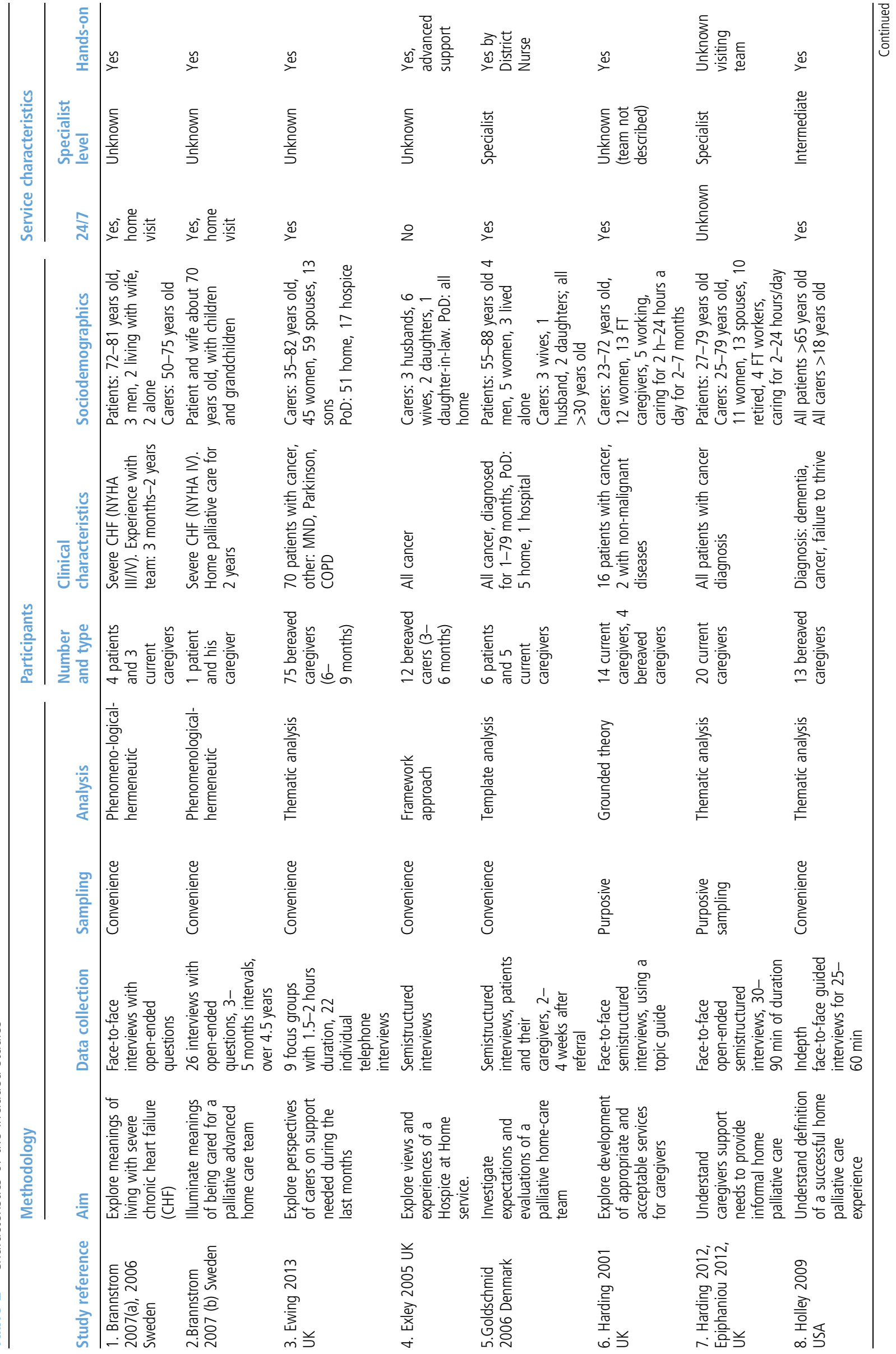


When visiting at home, palliative care professionals relieved caregivers from responsibility, by providing an assessment of the patient's situation and helping with decision-making, but also by helping with practical hands-on care (table 4, Q5-Q9). When the team had the resources to provide advanced technological support at home, hospital visits were avoided and feelings of security were enhanced. Home visits were also seen as an opportunity to listen to patients and families, to give information and to discuss advanced care planning. An unhurried calm attitude was seen as beneficial (table 4, Q10). Studies 3 and 9 identified the importance of the team being available to visit at the time of death (table 4, Q11-Q12).

Home visits were also seen as an opportunity to have a break from caregiving, hence relieving the caregiver by providing some form of respite. The need for formal structured respite services was not congruent among participants (table 4, Q15-Q16).

Competence

A team which was described by patients and caregivers as competent was able to prevent, manage and relieve suffering by providing effective physical and psychological symptom control and skilful communication. When relieved from suffering, participants reported enhanced feelings of security with opportunity to pursue other goals, mainly living family life at home with normality (ie, retaining family members' roles and usual family activities) and preparing for death.

Having access to knowledgeable professionals, who were able to relieve patients' physical and psychological symptoms, inspired trust in the team and hope in future symptom control (Q17-Q18, table 4).

The two-way process of communication was an essential component of home palliative care. Skilful communication facilitated the provision of individualised care adapted to family life, therefore enabling patients and caregivers to retain some degree of normality at home. A team that was available to listen to patients' and caregivers' life and disease history nonjudgmentally, and acknowledge their roles in the disease process but also in the family, was seen as supportive of patient and caregiver (table 4, Q19-Q21). Informing and giving anticipatory guidance was another important aspect of this component, as it allowed for patients and caregivers to participate in decision-making, increasing the chances of receiving care tailored to their specific needs and wishes, and adapted to family life (table 4, Q22-Q24). When caregivers were confident in their skills, care was better adapted to family life, enhancing the sense of normality and management of uncertainty (table 4, Q25).

\section{Line-of-arguments synthesis: security as the central} concept of home palliative care

When mapping and reflecting on the overall findings of the reciprocal translational synthesis (table 4), we 


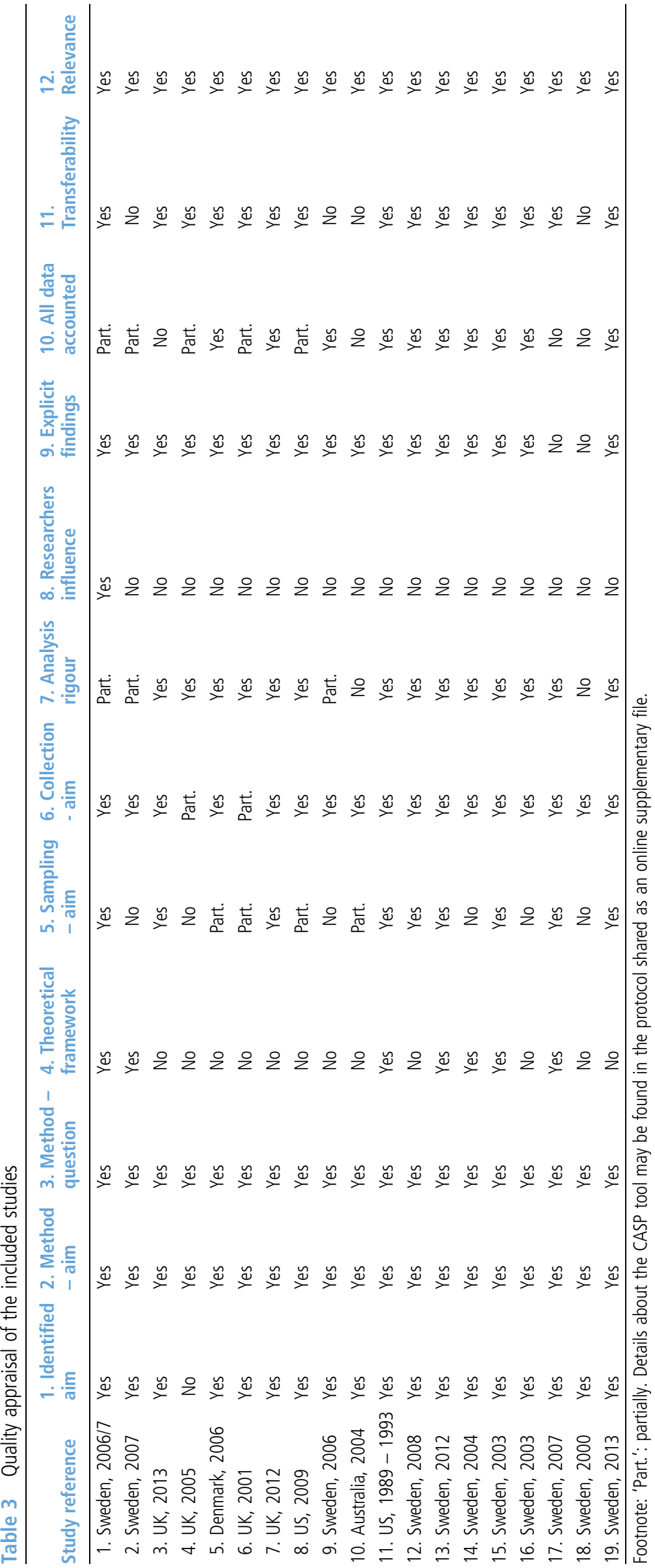




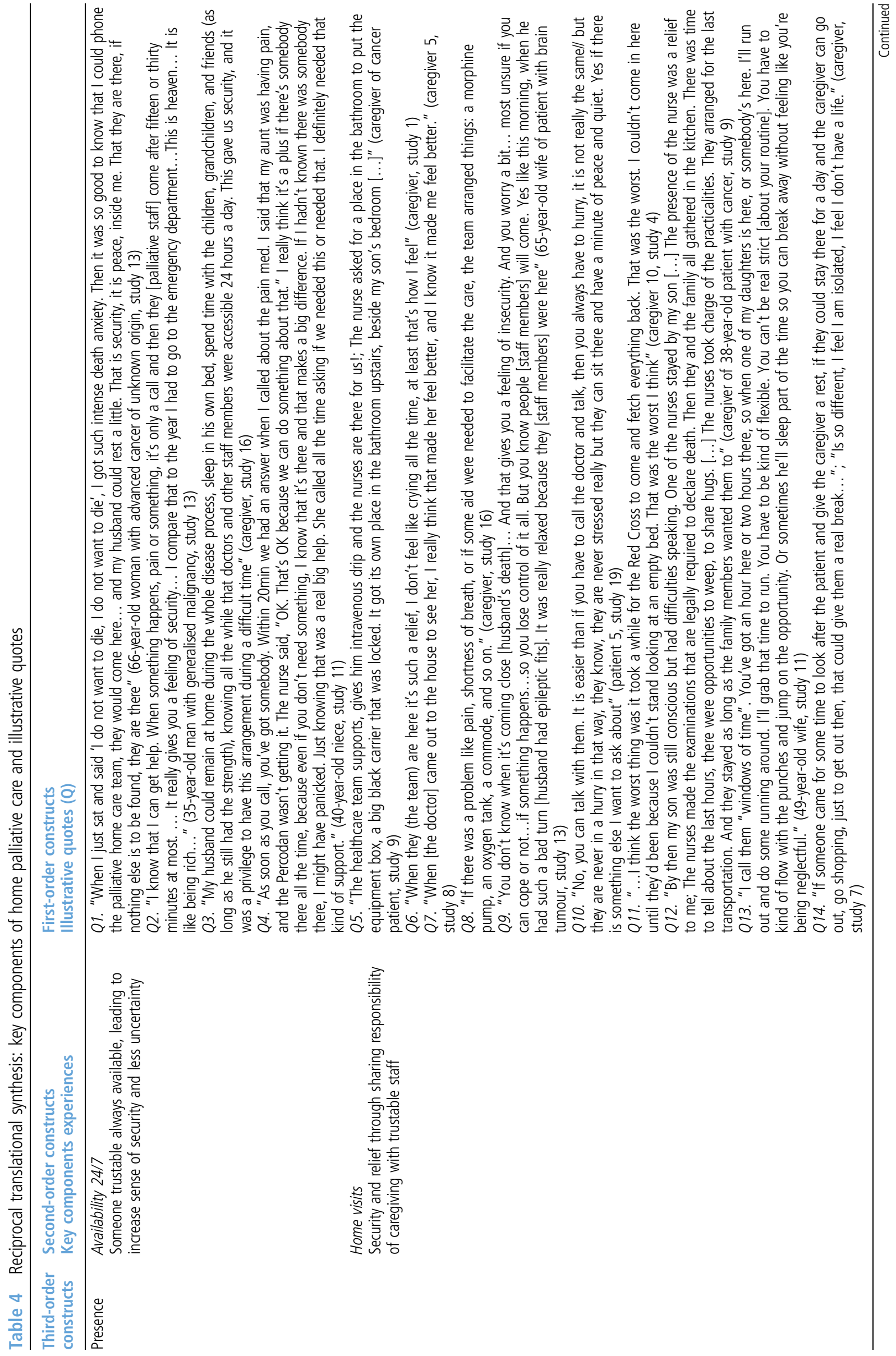



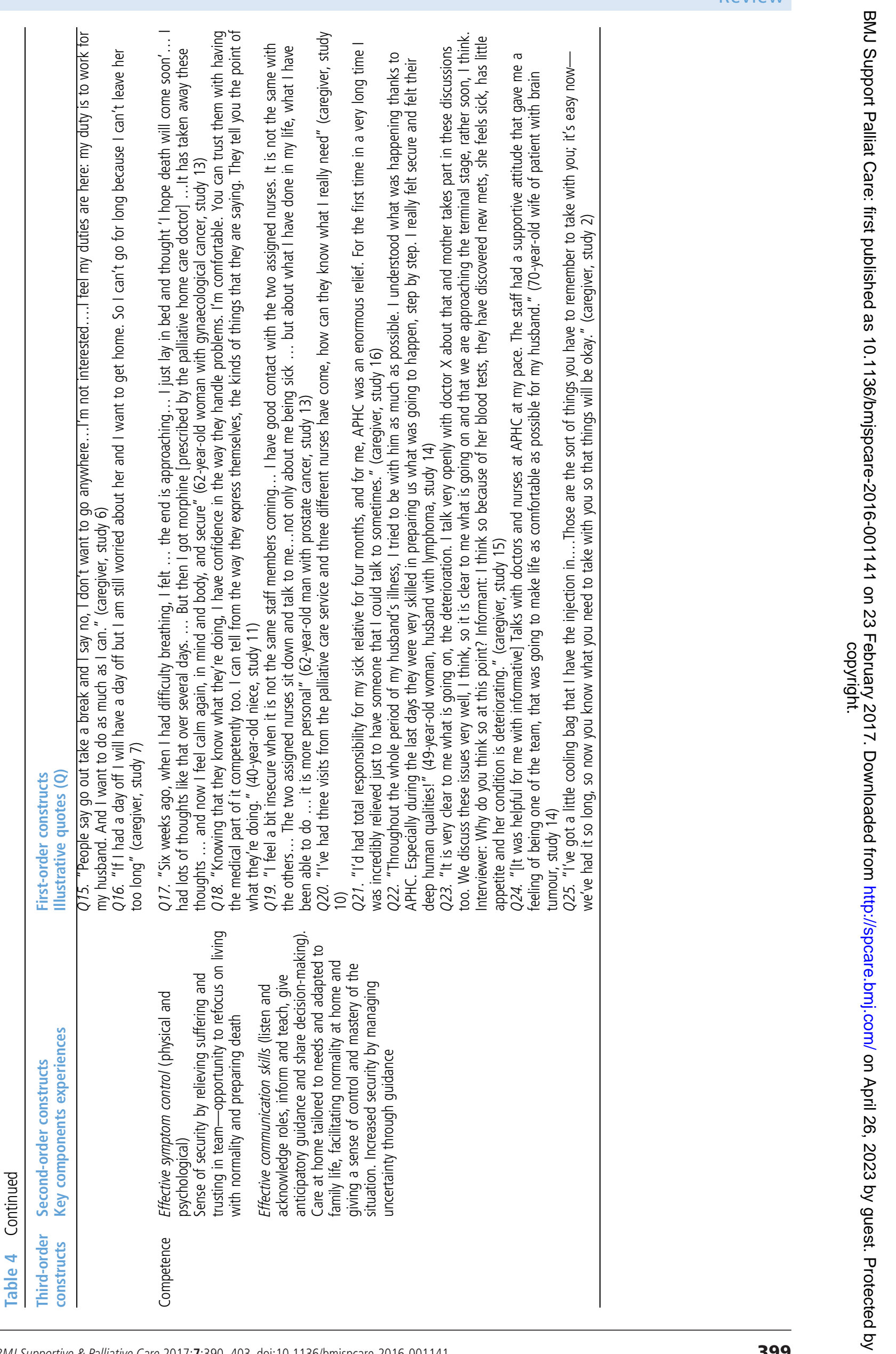
obtained a different level of interpretation, given that the four components contribute to some extent to the feeling of security at home (Q1-Q4, Q9, Q17-Q19, Q22), as shown by the second-order constructs (table 4). Security was therefore identified as the home palliative care core mechanism of action, enabling patients to stay at home, as illustrated by the following quote:

Now I feel safe if something happens. I know I can turn to you. (patient with cancer, Study 5)

An appropriate level of security seemed to be achieved when patients and/or caregivers were not under unacceptable levels of suffering, that is, when they felt relieved (from either burden or symptoms). Hence, when being cared for by a present team, participants felt that there was always someone available for support and sharing of responsibility. When the team was seen as providing competent care, it meant that the support available was trustworthy and effective (third-order constructs in table 4).

In turn, feeling secure allowed patients and caregivers to pursue two main valuable goals: (1) living family life, which was fostered by being cared for at home while preserving the most valuable experiences (sometimes akin to 'quality of life' or 'well-being'); (2) preparing for death, that is, saying goodbyes, completing the narrative, finding meaning. The following four quotes illustrate how home palliative care allowed for participants to pursue these meaningful goals, by contributing to their feeling of security at home.

He gets such good care and we are really happy. I also think the feeling of security makes us feel more free so we can go places we haven't been able to visit for years. (current caregiver of chronic heart failure patient, Study 1)

I perceived powerlessness when my husband, during his illness, did not get enough attention and care from healthcare. Since APHC came into our lives the powerlessness has changed to confidence and calm. There is time, both for my husband and me, to reflect and to some extent accept the difficult situation we are in. (63-year-old wife of patient with GI cancer, Study 14)

My husband could remain at home during the whole disease process, sleep in his own bed, spend time with the children, grandchildren, and friends (as long as he still had the strength), knowing all the while that doctors and other staff members were accessible 24 hours a day. This gave us security, and it was a privilege to have this arrangement during a difficult time. (caregiver, Study 17)

Interviewer: "Could you describe what it is like to feel secure?" Patient: "Life with the disease does not take over everything.... I know that if something is not working, I only have to make a phone call [to the palliative home care team] to get help. This makes you focus on other things. When I come home I do not think about it [the illness] at all ... I do other things. $\ldots$ and ie, the best praise I can give ... that I do not think about it.'” (35-year-old man with generalised malignancy, Study 13)

These relationships between key components, security, living life and preparing for death are depicted in a simplified conceptual model of the experiences of patients and caregivers with home palliative care (figure 1). According to the interpretation obtained in this review, we propose the following definition of security in this context: security means to be able to trust the home palliative care team to be there for support, prevention and relief of avoidable suffering at home, as the disease progresses.

\section{DISCUSSION}

By systematically reviewing the existing qualitative research on the experiences of patients and family caregivers with home palliative care services, this meta-ethnography found evidence that these specialist/intermediate teams have a major role in providing security at home. This was in turn achieved by the teams when they were seen as: (1) being present, that is, with availability to be contacted $24 / 7$ and to visit patients and caregivers at home, and (2) being competent, that is, providing effective symptom control and communicating skilfully. This interpretation of the patients and caregivers experiences implies that, by contributing to their sense of security, the home palliative care teams enabled patients and caregivers to focus on the dual process of living family life and preparing for death, while remaining at home.

Recent research suggests that caregivers' sense of security was higher when there was a trustful relationship with the physician, and when patients were relieved from physical distress. ${ }^{26}$ In a multivariable analysis, Milberg $\mathrm{et}_{\mathrm{al}} \mathrm{l}^{27}$ found that a sense of mastery over the situation was positively related to a higher sense of security.

The finding that patients and caregivers' sense of security is a condition for them to remain at home, creating the opportunity to pursue other goals, partially fits with Zalenski's model of Maslow's Theory of Needs (adapted to palliative care). In this model, safety is physical and emotional and refers to being free of fear. ${ }^{28}$ Also, these findings are aligned with the attachment theory ${ }^{29} 30$ in the sense that feeling secure enabled patients and caregivers to focus on goals with an exploratory nature, that is, living life and preparing for death. It is possible that these two processes configure a dynamic model, with patients and caregivers oscillating from one aspect to the other, as in the dual model of confrontation and avoidance behaviours when coping with bereavement. ${ }^{31}$ In fact, it is reasonable to assume that patients and caregivers are going through a process of anticipatory grief during the palliative care phase. ${ }^{32}$ 


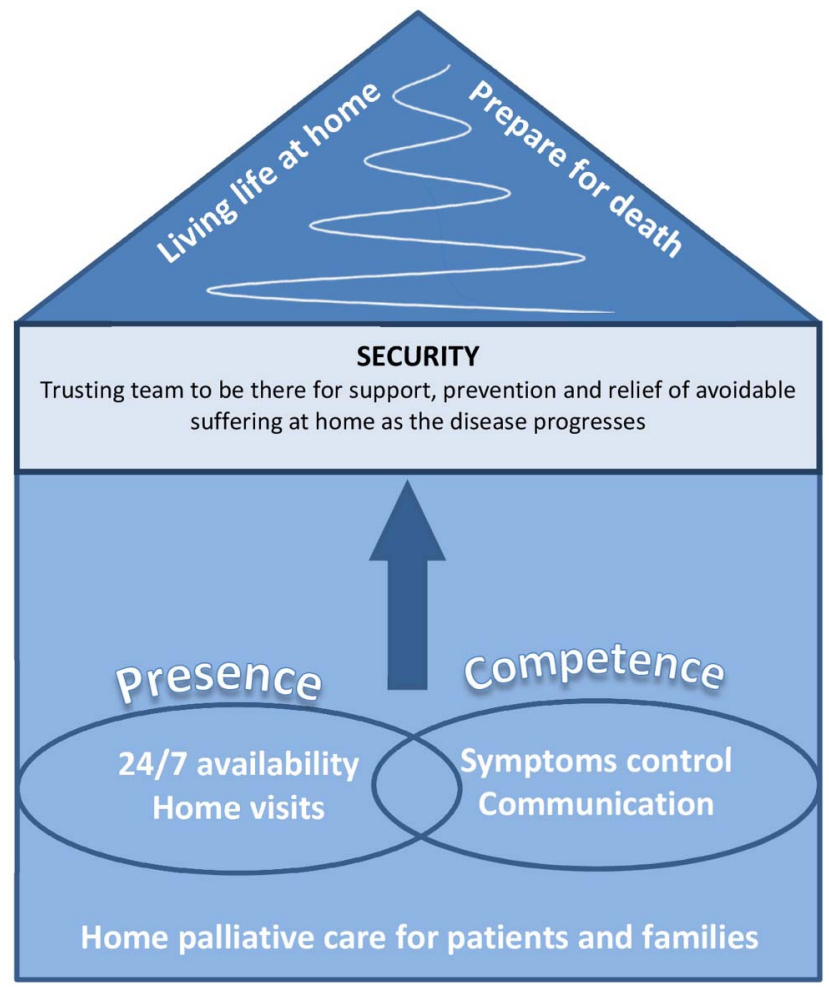

Figure 1 Lines-of-argument synthesis: simplified model of the experiences of patients' and caregivers' with home palliative care.

\section{Implications for policymakers, clinicians and future research}

Healthcare professionals taking care of these populations should be aware of their fundamental role in influencing the experience of security at home. Through competent skilful communication, teams provide individualised care adapted to family life, hence enhancing feelings of normality at home. Skilful communication is a complex intervention in itself, comprising: (1) listening to concerns with an open attitude; (2) acknowledging patients and caregivers as recipients and participants in care, and also family members; (3) informing, teaching, giving anticipatory guidance and facilitating shared decision-making.

This review also adds to the evidence that 24/7 availability is crucial for patients and caregivers to be cared for at home in such difficult situations, supporting the recommendation of the European Association of Palliative Care ${ }^{4}$ for teams to be available for contact at any time.

As being relieved from symptom burden is such an important experience in enabling security, teams should be able to screen and closely monitor symptom control, using for instance, patient-reported outcome measures like the Palliative care Outcome Scale (POS and POS-S). ${ }^{33}$ Finally, our review shows that caregivers experience visits by home palliative care teams to be a source of relief from caregiving burden. Professionals should be aware of this potential when visiting at home.

Despite security being central to the experiences of patients and caregivers, the more common measures used in the home palliative care context do not capture this concept. We therefore recommend that the role of home palliative care teams in enabling the experience of security should be better understood, and security should be considered as a potential outcome of these interventions. Also, integration of our results with the quantitative findings from the Cochrane review ${ }^{8}$ could further enlighten the heterogeneity found in the quantitative review, while contributing to the identification of these components as the active ingredients of home palliative care. Finally, researchers should be aware of the need to adequately report the home palliative care interventions received by participants of qualitative and quantitative studies.

\section{Strengths and limitations-review trustworthiness}

We used a systematic methodology to screen the published and unpublished literature according to the recommendations of the PRISMA statement, ${ }^{16}$ enhancing its dependability and credibility. ${ }^{34} 35$ The review feasibility and usefulness was tested during the pilot, and the limitations found were addressed at that stage. The review also shows transferability and confirmability, given that the 19 studies originated from 5 countries in 3 continents, and reported similar experiences of patients and caregivers with home palliative care services.

Being a structured interpretative methodology successfully used before in healthcare research, ${ }^{20}$ the meta-ethnographic method proved to be appropriate to meet the aim, guiding and structuring the interpretation of the findings, and allowing for interaction between phases.

Nonetheless, a meta-ethnography is a challenging methodology in all its stages. ${ }^{19} 20$

Despite the invaluable support from the library services and attempts to contact the authors, we could not retrieve seven references. Also, some decisions regarding inclusion criteria were made later in the review process and involved changes to the protocol (these are presented in an online supplementary file).

Owing to the study interpretative nature, the findings of this review are open to criticism. Other researchers with different philosophical stances and professional experiences could have obtained a different synthesis. The major a priori assumption of the review was that, by understanding the experiences of patients and caregivers with home palliative care, we would be able to identify which components of the interventions had a major role in shaping these experiences. To ensure that different perspectives were accounted for, and that the interpretation obtained reflected the results of the studies more than the views of the reviewers, this study involved: (1) 
establishing a review team with complementary professional experiences; (2) different reviewers developing separate syntheses; (3) developing a final conceptual model that encompassed the different interpretations and focused on the core findings of each reviewer.

\section{CONCLUSION}

This review showed that home palliative care increases the sense of security of patients and caregivers facing life-threatening diseases with palliative care needs at home. Professionals taking care of these populations should be aware of their security-enabling role, by providing competent care and being present. Home palliative care teams should be widely available and empowered with the resources to be competent (ie, providing effective symptom control and skilful communication) and present (offering 24/7 availability and home visits).

\section{Twitter Follow Vera Sarmento @ Vera P Sarmento}

Acknowledgements The authors express their gratitude to the Calouste Gulbenkian Foundation for funding the DINAMO project. The authors are most grateful to Mark Kluzek and the Interlibrary and Document Delivery team of King's College London for invaluable support in retrieving manuscripts for full-text screening; authors of the included studies, who provided the information asked for; colleagues in the Cicely Saunders Institute, mainly Bárbara Antunes and Jonathan Koffman for support and internal peer-review.

Contributors VPS designed and piloted the protocol under BG and IJH supervision. VPS identified and screened the studies, recorded and analysed the data, and wrote the report. MG participated in the screening and analyses, was closely involved in all steps and guided the interpretation process. $\mathrm{IJH}$ assessed the feasibility and usefulness of the review, with a major contribution during the protocol development and report of the results. BG directly supervised all steps of the review from its inception, provided the classification criteria for the levels of specialisation of home palliative care services and was involved in screening and analyses discussions. All authors contributed to this manuscript and agreed on the final version.

Funding This study was funded by the Calouste Gulbenkian Foundation in the context of the DINAMO Project. DINAMO aims to enhance advanced training and research to optimise home palliative care in Portugal. Principal investigator: BG. Scientific director: IJH. Other members of DINAMO: Ana Lacerda, Catarina Ribeiro, Diogo Martins-Branco, Duarte Soares, Helder Aguiar, Maja de Brito, Pedro L. Ferreira, Rita Canario and VPS. IJH is an NIHR Senior Investigator and Head of Department of Palliative Care, Policy and Rehabilitation at King's College London.

Competing interests None declared.

Provenance and peer review Not commissioned; externally peer reviewed.

Data sharing statement A table of the full reciprocal translation with participants' quotes and explanations offered by the authors to illustrate the interpretation and identification of the key components as such is available as WDS. The Excel file with the recording table is available on request from the corresponding author at verapsarmento@gmail.com.

Open Access This is an Open Access article distributed in accordance with the Creative Commons Attribution Non Commercial (CC BY-NC 4.0) license, which permits others to distribute, remix, adapt, build upon this work non-commercially, and license their derivative works on different terms, provided the original work is properly cited and the use is non-

commercial. See: http://creativecommons.org/licenses/by-nc/4.0/

\section{REFERENCES}

1 UN General Assembly, Universal Declaration of Human Rights, 10 December 1948, 217 A (III), available at: http://www. refworld.org/docid/3ae6b3712c.html (accessed 13 Feb 2016).

2 Connor SR, Bermedo MCS, eds. Global atlas of palliative care at the end of life. World Palliative Care Alliance \& World Health Organisation, 2014.

3 Gomes B, Calanzani N, Gysels M, et al. Heterogeneity and changes in preferences for dying at home: a systematic review. BMC Palliat Care 2013;12:7.

4 European Association for Palliative Care (EAPC). White Paper on standards and norms for hospice and palliative care in Europe: recommendations from the European Association for Palliative Care. Eur J Palliat Care 2009;16:278-89.

5 Gomes B, Cohen J, Deliens L, et al. International trends in circumstances of death and dying. In Gott M, Ingleton C, (eds). Living with ageing and dying: palliative and end of life care for older people. Oxford: Oxford University Press; 2011. pp. 3-18.

6 Jordhoy MS, Fayers P, Saltnes T, et al. A palliative-care intervention and death at home: a cluster randomised trial. Lancet 2000;356:888-93.

7 World Health Organisation Executive Board. Resolution EB134.R7: strengthening of palliative care as a component of integrated treatment within the continuum of care. 2014. http://apps.who.int/gb/ebwha/pdf_files/EB134/B134_R7-en.pdf

8 Gomes B, Calanzani N, Curiale V, et al. Effectiveness and cost-effectiveness of home palliative care services for adults with advanced illness and their caregivers. Cochrane Database Syst Rev 2013;6:CD007760.

9 Centeno C, Lynch T, Donea O, et al. EAPC Atlas of Palliative Care in Europe 2013. Full Edition. Milan: EAPC Press; 2013.

10 Melin-Johansson C, Henoch I, Strang S, et al. Living in the presence of death: an integrative literature review of relatives' important existential concerns when caring for a severely ill family member. Open Nurs J 2012;6:1-12.

11 Wilkinson E, Salisbury C, Bosanquet N, et al. Patient and carer preference for, and satisfaction with, specialist models of palliative care: a systematic literature review. Palliat Med 1999;13:197-216.

12 France EF, Ring N, Noyes J, et al. Protocol-developing meta-ethnography reporting guidelines (eMERGe). BMC Med Res Methodol 2015;15:1-14.

13 Craig P, Dieppe P, Macintyre S, et al. Developing and evaluating complex interventions: the new Medical Research Council guidance. BMJ 2008;337:a1655.

14 Evans CJ, Harding R, Higginson IJ, MORECare. 'Best practice' in developing and evaluating palliative and end-of-life care services: a meta-synthesis of research methods for the MORECare project. Palliat Med 2013;27:885-98.

15 Petticrew M, Anderson L, Elder R, et al. Complex interventions and their implications for systematic reviews: a pragmatic approach. J Clin Epidemiol 2013;66:1209-14.

16 Liberati A, Altman DG, Tetzlaff J, et al. The PRISMA statement for reporting systematic reviews and meta-analyses of studies that evaluate health care interventions: explanation and elaboration. Ann Intern Med 2009;151:W65-94.

17 Noblit GW, Hare RD. Meta-ethnography: synthesizing qualitative studies. Sage, 1988. 
18 Campbell R, Pound P, Pope C, et al. Evaluating metaethnography: a synthesis of qualitative research on lay experiences of diabetes and diabetes care. Soc Sci Med 2003;56:671-84.

19 Campbell R, Pound P, Morgan M, et al. Evaluating meta-ethnography: systematic analysis and synthesis of qualitative research. Prepress Projects Limited, 2011.

20 Britten N, Campbell R, Pope C, et al. Using meta ethnography to synthesise qualitative research: a worked example. J Health Serv Res Policy 2002;7:209-15.

21 Walters LA, Wilczynski NL, Haynes RB. Developing optimal search strategies for retrieving clinically relevant qualitative studies in EMBASE. Qual Health Res 2006;16:162-8.

22 Campbell M, Fitzpatrick R, Haines A, et al. Framework for design and evaluation of complex interventions to improve health. BMJ 2000;321:694.

23 Donabedian A. Quality assessment and assurance: unity of purpose, diversity of means. Inquiry 1988;25:173-92.

24 Critical Appraisal Skills Programme. 10 questions to help you make sense of qualitative research. http://www.casp-uk.net/ wp-content/uploads/2011/11/CASP_Qualitative_Appraisal_ Checklist_14oct10.pdf (accessed 10 Oct 2013).

25 Dixon-Woods M, Sutton A, Shaw R, et al. Appraising qualitative research for inclusion in systematic reviews: a quantitative and qualitative comparison of three methods. J Health Serv Res Policy 2007;12:42-7.
26 Igarashi A, Miyashita M, Morita T, et al. Association between bereaved families' sense of security and their experience of death in cancer patients: cross-sectional population-based study. J Pain Symptom Manage 2016;51:926-32.

27 Milberg A, Friedrichsen M, Jakobsson M, et al. Patients' sense of security during palliative care-what are the influencing factors? J Pain Symptom Manage 2014;48:45-5.

28 Zalenski RJ, Raspa R. Maslow's hierarchy of needs: a framework for achieving human potential in hospice. J Palliat Med 2006;9:1120-7.

29 Bowlby J. Attachment and loss v. 3. Random House, 1969.

30 Loetz C, Müller J, Frick E, et al. Attachment theory and spirituality: two threads converging in palliative care? Evid Based Complement Alternat Med 2013;2013:740291.

31 Stroebe M, Schut H. The dual process model of coping with bereavement: rationale and description. Death Stud 1999;23:197-224

32 Zeitlin SV. Grief and bereavement. Prim Care 2001;28:415-25.

33 Bausewein C, Le Grice C, Simon S, et al. The use of two common palliative outcome measures in clinical care and research: a systematic review of POS and STAS. Palliat Med 2011;25:304-13.

34 Guba EG. Criteria for assessing the trustworthiness of naturalistic inquiries. ECTJ 1981;29:75-91.

35 Krefting L. Rigor in qualitative research: the assessment of trustworthiness. Am J Occup Ther 1991;45:214-22. 